\title{
Lambda Z Time Upper Limit
}

National Cancer Institute

\section{Source}

National Cancer Institute. Lambda Z Time Upper Limit. NCI Thesaurus. Code C85654.

The upper limit on timing values to be included in the calculation of Lambda $z$. 